\title{
Teoría económica y Estado del Bienestar. Una aproximación
}

Fernando Salazar Silva ${ }^{1}$

Recibido: Octubre 2005

Magíster en Estudios Políticos de la Universidad Javeriana, Ph.D (c) en economía de la Universidad de Sevilla, economista de la Universidad del Valle. Docente investigador Departamento de Economía Pontificia Universidad Javeriana Cali.

Aceptado: Febrero 2006 


\section{Introducción}

El Estado de Bienestar, proceso institucional de gran recorrido histórico, es objeto en las décadas recientes de un arduo debate en torno a las implicaciones de sus funciones en la cohesión social, lo cual amerita que se esboce algunas claves interpretativas para la discusión.

Este artículo, que procura brindar una serie de elementos para la reflexión, se estructura en dos epígrafes. En el primero, se abordan las unidades mínimas del Estado del Bienestar lo que se ha denominado clásico. El segundo se bosqueja una reflexión de la teoría económica.

\section{El Estado de bienestar clásico}

El Estado de bienestar es el punto de convergencia entre el objetivo de primer orden (el desarrollo del mercado) y el de segundo orden (concepción protectora). Esta nueva forma de concebir el orden social se apoya en tres principios básicos. El contrato social, el mercado autorregulado y el seguro (González Seara, 2000, p. 119).

El resultado teórico de Europa se localiza en la concepción de Keynes expresada en su "Teoría General de la ocupación, el interés y el dinero", publicada en $1936^{2}$. La postura de Keynes concedió una ruptura con el en- foque teórico clásico, al no poder explicar esta última los hechos reales (Keynes, 1991, p. 15).

El punto de atención gubernamental giraba alrededor del problema de la distorsión del mercado laboral (alta tasa de desempleo), y Keynes lo relacionó con la deficiencia en la demanda efectiva, como consecuencia de los bajos niveles de consumo e inversión productiva. Bajo este panorama, la actuación del Estado permitiría que el gasto público influyera, por un lado, de manera directa en el consumo y la inversión, y por otro, el uso de las políticas fiscales y monetarias. En Keynes, pues, se encuentra su objeto teórico que ratifica la intervención del Estado, sin que la intervención quede establecida de forma explícita por la doctrina keynesiana.

\section{Momentos que favorecen el origen y la consolidación de los Estados de bienestar}

El profesor Muñoz de Bustillo (2000) considera cuatro elementos para el origen y consolidación de los Estados de Bienestar. En primer lugar, la revolución industrial, que expresa la ruptura con el orden social vigente y su cohesión social y el fortalecimiento del mercado como expresión de la relación social; en segundo lugar, la construcción de propuestas sociales y políticas reales, alternativas al capitalismo; en tercer lugar, la presencia de la

2 La primera edición inglesa de 1936 se titulaba The General Theory of Employment, Interest and Money. En el presente artículo se maneja la editada por el Fondo de Cultura Económica del año 1991. 
ideología que se fundamenta en el principio político de sostener el Bienestar Social, y por último, la aceptación de la intervención estatal presente en la obra de Keynes.

\section{La revolución industrial}

El profesor Muñoz de Bustillo (2000, pp. 20) argumenta que el capitalismo favorece la dependencia de los individuos de las decisiones de otros, situación que origina una ampliación de su inseguridad y su vulnerabilidad económica ${ }^{3}$. Considera además que la Revolución Industrial deteriora las formas de seguridad forjados en las sociedades preindustriales ${ }^{4}$, lo que se traduce en un desarraigo de los viejos esquemas de sujeción y un encuentro de incertidumbre con las nuevas representaciones de organización social ${ }^{5}$
Es pertinente hacer notar que la revolución industrial, en la línea del profesor, (Muñoz de Bustillos, 2000, p 22) deteriora las redes de seguridad y por ende el bienestar de los trabajadores; tal deterioro se acentúa aún más cuando no se acompaña de un crecimiento del ingreso y su impacto en las condiciones de vida material de los trabajadores. Situación que hubiese permitido la constitución de mecanismos alternativos de seguridad:

"la revolución industrial tiene una primera fase que cubriría grosso modo el último cuarto del siglo XVIII y la primera mitad del siglo XIX, en donde el impacto sobre el nivel de vida de los trabajadores muy probablemente fuera negativo, aunque sin llegar a "niveles asiáticos", para, a partir de entonces, empezar una senda creciente hasta nuestros días" (Muñoz de Bustillo, 2000, p. 23).

3 Los instrumentos de atención social constituidos en el siglo XVI con la ley de los pobres, se resquebrajaron por los intereses del liberalismo económico del siglo XVIII. Aparece por ejemplo la renta mínima en forma de abono salarial, como elemento constitutivo de protección social (Polanyi, 1989, p. 151)

$4 \quad$ Las leyes de los pobres responden a los desarreglos del mercado, en este sentido manifiesta Trevelyan $(1946,246)$ que más de un millón de personas, es decir, la quinta parte de la población total de la nación (Inglaterra), recibían limosna ocasionalmente, la mayoría en forma de auxilio pagado por las parroquias.

Para el caso de Alemania de Bismarck, en otro momento histórico, se discutía el control de los trabajadores por parte del Estado no sólo a través de la represión sino a través de medidas que favorezcan y dividan a los trabajadores (en Navarro, 2000, p. 76).

Para ampliar el caso de la constitución del Estado de Bienestar en Alemania véase Gómez de Pedro (2002, capítulo III, p. 217- 236)

5 En esta misma línea argumentativa se ubica Ashford cuando manifiesta que el primer capítulo de la aparición de los Estado de Bienestar tuvo como objetivo modificar las reglas de juego de ayuda caritativa o providencial (1989, p. 345).

Una reflexión detallada de carácter histórico-teórico sobre las condiciones de precariedad social en Inglaterra lo hace Villarespe (2002). En el texto aparece tratado de manera seria el trabajo de Webb, S y Webb B. 
Sobre la distribución de la renta, la revolución provoca de manera generalizada en las economías europeas altos grados de desigualdad durante el siglo XIX. Si bien hay un impacto sobre el ingreso, también tiene efectos sobre las condiciones de vida de los individuos, al no crearse de manera adecuada acceso a bienes como la salud, la educación, entre otros.

Frente a esta situación, se da comienzo a la presión social en términos de la creciente demanda de protección social, lo cual desemboca en la institucionalización de las mismas. Aparecen movimientos sindicales y de ciudadanía que protagonizan papel preponderante en la constitución del Estado de Bienestar (Muñoz de Bustillo, 2000, p. 25).

\section{Movimientos alternativos}

La dinámica del mercado si bien generó distorsiones en las condiciones de vida, las soluciones a partir de la gestión pública no logró conseguir resultados que minimizaran el descontento social, esto se agudizó en la medida que la ausencia de una alternativa política distinta al mercado no consiguió consolidarse.

La aparición de la militancia sindical llevó al Estado a diseñar mecanismos para incorporar el descontento de los trabajadores a su interés. Los distintos gobiernos consideraron perti- nente la intervención creciente en los asuntos laborales, procurando con ello, la estabilidad de la eficacia y eficacia del sistema (Muñoz de Bustillo, 2000, p. 28).

La importante presión del movimiento obrero en el diseño de políticas sociales, crea la oportunidad para que el Estado asuma la protección social y garantice la legitimidad de la institucionalidad. La aparición de estos movimientos supone la génesis de estrategias alternativas a las del mercado, sin embargo, representan políticas compensatorias por parte del Estado, debido a que los grupos de trabajadores quedan inscritos dentro de la lógica del capital:

"La corrección por el Estado de los efectos disfuncionales de la sociedad industrial competitiva, no sólo es una exigencia ética, sino también, una necesidad histórica, pues hay que optar necesariamente entre la revolución y las reformas sociales"(Garcia-Pelayo, 1991, p. 353) .

Si bien la literatura que hace referencia al Estado de Bienestar pone de relieve en la reforma social como mecanismo para contener los partidos socialistas, no hay que desdeñar que detrás de las reformas se encuentra un propósito político consistente en adecuar a la "nueva" masa de trabajadores a la lógica del sistema económico ${ }^{7}$.

6 Podría ampliarse con detalle este punto a partir del tratamiento que esboza Dolleans $(1969,28)$

7 Con referencia a Hayek (1985) no es explicable la confrontación del discurso liberal ortodoxo a las reformas sociales con la posición del exceso de paternalismo del Estado de Bienestar. 


\section{Componente ideológico}

Uno de los aspectos que llama la atención en la configuración del Estado de Bienestar, después de la segunda guerra mundial, es la importancia del elemento ideológico del partido de gobierno.

El profesor Muñoz de Bustillo (2000, pp. 30-33) muestra que la relación ideología-Estado de Bienestar presenta una serie de contradicciones desde comienzos del siglo XX hasta entrados los años setenta (70). En algunos momentos parece que hay un efecto marginal entre la postura ideológica y la consolidación del Estado de Bienestar, pero en otros se evidencia una fuerte relación entre ambas al explicarse las altas tasas de transferencias.

"Habrá que esperar a la aparición de las primeras críticas al Estado de Bienestar, tras el desencadenamiento de las crisis económicas de los setenta, para que la ideología pase a tener un papel importante a la hora de explicar el grado de beligerancia con respecto a la implicación pública en la solución de los problemas de bienestar social" (Muñoz de Bustillo, (2000, p. 32).

El cambio de interpretación del funcionamiento agregado de la economía de mercado y del papel del Estado.

Cuando se hace referencia a los pilares del Estado de Bienestar, se toma como principio la teoría económica keynesiana, y su aplicación en Europa a través del Plan Marshall

A partir del aporte de Adam Smith, recogido en la Riqueza de las Naciones, las premisas de acción de la Hacienda Pública giraron alrededor de los elementos de economía política de libre mercado ${ }^{8}$. A partir de mediados del siglo XX se presentó un cambio dirigido a la intervención del Estado.

El cambio del modelo teórico, para comprender el funcionamiento del sistema capitalista, podría parcialmente ser explicado a través de un suceso histórico, el crack de la bolsa de Nueva York, el 11 de septiembre de 1929 y la subsiguiente crisis de los años $30^{9}$.

Keynes ${ }^{10}$ brinda una exégesis alternativa del funcionamiento de la economía, en la que el desempleo constituye un problema inherente al capitalismo, y esboza una justificación teórica a la intervención del Estado con el objeto de contrarrestar el desempleo. De manera categórica ofrece una solución de orden práctica a un problema concreto, a través de una interpreta-

8 En el primer capítulo del libro V Smith precisa los espacios de la actividad económica en los que se legitima la intervención del Estado: defensa, justicia, gastos de mantenimiento de la dignidad del soberano y gastos de obras e instituciones públicas.

$9 \quad$ Véase el clásico libro sobre el Crack del 29 de J.K. Galbraith (1974).

10 El punto neurálgico de la obra de Keynes es la explicación de la no vigencia de la Ley de Say. Keynes atribuye a la demanda el papel activo en la economía. Ahora bien, hace énfasis en el gasto público como variable operativa. Tal como lo esgrime Guerrero (1997, p. 30) Keynes es un liberal coherente con el sistema y esto no le es un obstáculo para recurrir a la intervención del Estado para salvar el capitalismo. 
ción teórica distinta a la del modelo clásico o neoclásico, tal como lo manifiesta Muñoz de Bustillo (2000, p.38):

"el análisis keynesiano conduce a un cambio radical en la interpretación del Estado como agente económico, ya que a las "funciones clásicas" se le suma la de actuar, mediante la gestión de la demanda efectiva, como garante del pleno empleo".

A partir de la publicación de la Teoría General se da comienzo a una nueva base teórica para poder soportar el Estado de Bienestar. Es conveniente aclarar que si bien el Estado de Bienestar y el keynesiano son diferentes, el primero tiene el fundamento práctico y doctrinal de la Teoría General.

\section{Los pilares del Estado de bienestar}

El Estado de Bienestar se puede particularizar a partir de lo que se conoce como el "Pacto Keynesiano" (Anisi, 1989), el cual tiene como objeto establecer la cohesión social a través de la incorporación de los trabajadores al crecimiento económico ${ }^{11}$, es decir, con esto se evita tasas de desempleo creciente. La absorción de mano de obra al proceso productivo es un elemento clave en la generación del crecimiento. El acuerdo deviene del hecho de la convergencia de intereses aparentemente contrapuestos, como premisa legitimadora de la reproducción del capital, tanto por parte de los trabajadores y el Estado:

"En resumen, el Estado de Bienestar pretendía hacer al capitalismo liberal económicamente más productivo y socialmente más justo. La prueba de ello, iba a ser su funcionamiento a lo largo del tiempo. El capitalismo de bienestar de la postguerra funcionó bien, tanto en términos económicos como sociales, que su éxito sirvió para respaldar los supuestos integradores recogidos en las recetas de Keynes y Beveridge (Mishra, 1992, p.33).

Otro elemento a considerar es el contenido social, el cual se garantiza desde el Estado a sus miembros a través de un conjunto de servicios sociales mínimos. Uno de los aportes del Estado de Bienestar, en este orden, es la nueva concepción de ciudadano, ahora con investidura de derechos los cuales van a ser garantizados ${ }^{12}$.

\section{El Pleno Empleo}

Entre las consideraciones que plasma Keynes en la Teoría General, una de ellas está dirigida a la eliminación del desempleo, la distorsión del funcionamiento más preocupante del mercado laboral. La comprensión del Estado de Bienestar se hace difícil con la

11 Este modelo de regulación del ciclo económico contiene un elemento de orden político, consistente en integrar por la vía política a la clase obrera. De esta manera se legitima el sistema de mercado.

12 La orientación del Estado en sus funciones económicas y políticas tiene impacto en el contexto constitucional al interior del Estado Social de Derecho. Cuyo objetivo es regular particularidades de la relación social. 
presencia de altas tasas de desempleo, debido precisamente a que el Estado tiene como objeto la sostenibilidad del mercado laboral ${ }^{13}$.

\section{Provisión universal pública de servicios sociales}

El conjunto de servicios sociales mínimos es concretado por el Estado a través de la provisión generalizada y pública de los servicios sociales, entre los que se puede contar, la salud, la educación y las pensiones.

Después de la Segunda Guerra Mundial, los Estados de Bienestar en Europa se pueden caracterizar por el modelo socialdemócrata, cuya premisa es la universalidad, una oferta creciente de servicios sociales de alta calidad, una presencia significativa de la producción pública, y la financiación a través de impuestos generales (Muñoz de Bustillo, 2000, p. 41).

\section{Actitud vigilante ante la marginación y la pobreza: garantía de un nivel de vida mínimo.}

Este es uno de los componentes del Estado de Bienestar que más atención ha recibido después de la Segunda Guerra Mundial. El objeto consiste en limitar situaciones de marginalidad y de pobreza $^{14}$ que pueden acabar la cohesión social del sistema, poniendo en ries- go la generación de valor económico. La reforma social de rentas mínimas y de prevención de la pobreza, en sus distintas dimensiones, han sido los baluartes del Estado de Bienestar clásico.

En los actuales momentos de transformaciones de la organización económica mundial, se está recrudeciendo la marginalidad y la pobreza, no encontrándose soluciones políticas por parte de los diferentes Estados, poniendo así en riesgo la estabilidad del sistema (Galbraith, 1992, p. 38). En definitiva, tres pilares que han sostenido con mayor o menor fortuna el Estado de Bienestar desde mediados del siglo $\mathrm{XX}$, pero que, ahora, en el arranque del nuevo siglo, parecen debilitarse sin remedio y sin que se atisbe un modelo alternativo de una solidez equivalente. El modelo que parece declinar se inscribe en lo que Bauman (2002, p.126) denomina "ética moderna" y "orden moderno" y deriva en su reforma a un "orden posmoderno". El cambio de un orden a otro viene caracterizado por pasar:

"de la regulación normativa a la seducción, de la planificación política a las relaciones públicas, de la obligación a la publicidad. Pienso que lo que sustenta todos estos cambios es el hecho de que los organismos centralizados y estrechamente administrados que se encargaban

13 Según Castell y Bosh (1998, p. 22) keynes asumía que el sistema capitalista sólo sobrevivir si se reformaba, y consideraba mecanismos de acuerdo entre los capitalistas y los trabajadores.

14 Muñoz de Bustillos (1989, p. 12) define la revolución keynesiana como un conjunto de actuaciones públicas tendientes a garantizar a todo ciudadano de una nación, por el mero hecho de serlo, el acceso a un mínimo de servicios que garanticen su supervivencia. 
de la tarea de la integración social la han cedido a las "fuerzas del mercado", fundamentalmente dispersas y descoordinadas".

Entramos, según Bauman, en un periodo posmoderno o mejor de " $m o$ dernidad líquida", definida como "la modernidad menos sus ilusiones" (Bauman, 2002, p. 106).

\section{La crisis del Estado del bienestar}

La propuesta de cohesión social a través del Estado de Bienestar, en su acepción socialdemócrata, sigue su curso a pesar del enfoque teórico neoclásico, si bien es cierto, que han empezado a ser redefinidas algunas de sus premisas, continua siendo de gran importancia para asegurar la generación y reproducción de capital. De los pilares que se han mencionado, el de provisión universal de los servicios sociales sigue persistiendo. Y no obstante, el crecimiento económico que se registra en la mayoría de los países de Europa, el desempleo, la pobreza y las desigualdades se evidencia de manera aguda. El contexto de la organización social actual actúa bajo un crecimiento que se puede considerar excluyente.

Hoy en día algunas teorías van ganando espacio en la explicación del nuevo modelo de Estado de Bienestar. Tal como lo manifiesta el profesor Muñoz de Bustillos, incluso no pueden ser todas del orden liberal ni mu- cho menos la presencia de una teoría hegemónica:

"No es sencillo resumir la esencia de ese pensamiento anti-keynesiano, en parte porque está formado por un conjunto muy distinto de propuestas teóricas, en algunos casos contrapuestas entre sí, que sin embargo comparten una misma evaluación (negativa) de la intervención pública, abogando por la vuelta a un estado residual que permita la libre actuación del mercado en el mayor número posible de campos de la actividad humana"(Muñoz de 2000, p 51).

El Estado de Bienestar en su concepción keynesiana establece una fuerte conexión entre los fundamentos económicos y la acción política ${ }^{15}$. Recuérdese que la Teoría General es un soporte económico para el propósito político de sostenibilidad del sistema capitalista:

"En verdad el mundo no tolerará por mucho tiempo más la desocupación que, aparte de breves intervalos de excitación, va unidad -y en mi opinión inevitableal capitalismo individualista de estos tiempos; pero puede ser posible que la enfermedad se cure por medio de un análisis adecuado del problema, conservando al mismo tiempo la eficiencia y la libertad" (Keynes, 1991, p. 335)

En las actuales circunstancias, la generación de desempleo y desigualdades puede ser explicada a través de la ausencia de consenso entre las premisas económicas y la práctica política.

15 Lo particular en Keynes fue haber establecido la relación entre el orden social y el desarrollo económico. Situación contraria a la planteada por la escuela liberal ortodoxa que asumía la igualdad como producto del desarrollo económico. 


\section{El fin del pacto keynesiano y la revolución neoconservadora}

Una de las preocupaciones en la dinámica del Estado de Bienestar actual, es el distanciamiento entre las distintas perspectivas de análisis de la ciencia económica. Los profesores Heilbroner y Milberg en su texto sobre "la crisis de visión en el pensamiento económico moderno"16 presentan una postura de gran interés frente a este tema. Las autores toman como punto de apoyo el concepto schumpeteriano de "situación clásica".

El aporte de los profesores gira alrededor de la ausencia en el contexto de la historia del pensamiento económico reciente de un interés común sobre las condiciones de la realidad económica y política. A diferencia de los modelos clásicos, neoclásicos y keynesianos los cuales tenían un interés común del mundo real, hoy en día, no podríamos referirnos con tanta certidumbre (Heilbroner y Milberg, $1998 \mathrm{p}$. 16). Otro elemento que consideran los autores es la falta de "visión" del pensamiento económico.

El contexto de crítica en el que se ubican los autores para mostrar esta ausencia de interés común alrededor del mundo real, la buscan en la forma como está siendo dirigida la teoría económica norteamericana:
"El signo de la economía de nuestros días es su extraordinaria indiferencia en relación a este problema -se refieren a la preocupación continua y visible por la conexión entre teoría y realidad-. En sus momentos álgidos, la "fuerte teorización" del presente periodo alcanza un grado de irrealidad que sólo se puede comparar con la escolástica medieval" (Heilbroner y Milberg, 1998, p. 18).

Los profesores Heilbroner y Milberg enfatizan que la ausencia a la que se han referido, puede replantearse si se considera como punto fundamental la visión del pensamiento económico, es decir su relación con el mundo real (esto evita tratar a la economía como mercado). Hoy en día se está más inclinado a la realización de análisis confundiéndose economía con éste, llegando a creer que el análisis económico puede existir como una cierta clase de estudio social desencarnado (Heilbroner y Milberg, 1998, p. 21).

Cuando se habla de la relación del pensamiento económico y el mundo real, se está haciendo referencia a una reflexión analítica de los problemas económicos apoyados en los "macrofundamentos" en donde lo económico va más allá del mercado, al respecto el profesor Anisi manifiesta:

"esa perspectiva económica en la que Jerarquía, Mercado y Valores se interrelacionan, excluyen, apoyan... permite contemplar de forma adecua-

16 El libro fue publicado en 1995 por Cambridge University Press. Su traducción al español la realizó Paidós en 1998. 
da aquello que es y fue el auténtico núcleo de la economía: las relaciones de poder" (1992, p 35).

El llamado de atención que se podría plantear es que en las actuales circunstancias la teoría económica no incorpora en sus estudios analíticos las contradicciones del sistema. En la medida que los conflictos sociales no sean reconocidos, la economía no podrá dar cumplimiento de su visión, es decir del estudio de las condiciones de la realidad humana.

Crisis de la ciencia económica y el Estado de Bienestar

Las formulaciones teóricas clásica, marginalista y keynesiana cumplían una función estabilizadora del sistema económico (Heilbroner y Milberg, 1998, p. 38), lo que se reflejaba para el caso del keynesianismo por ejemplo, en calmar las incertidumbres de los hombres con la configuración del Estado de Bienestar. Hoy en día, la teoría económica, como es concebida, no cumple esa función estabilizadora. Los economistas no dirigen la economía hacia la resolución de los conflictos sociales:

"el fracaso a la hora de alcanzar una nueva situación clásica es el resultado del giro equivocado, en términos de visión, que tomó el pensamiento económico cuando se enfrentó a una encrucijada a principios de la década de los años setenta" (Heilbroner y Milberg, 1998, p. 39).
Una de las razones que se puede esgrimir de esa ausencia de visión, puede atribuirse a la crisis que se dio en el año 1973 y que ha repercutido a lo largo del siglo XX en la ciencia económica (Anisi, 1995, p. 67). La crisis económica está relacionada con la pérdida de legitimidad ideológica del la propuesta keynesiana sobre el Estado de Bienestar, esto no significa que la crisis económica haya puesto en entredicho el Estado de Bienestar, sino que la puesta en entredicho del Estado de Bienestar fue la crisis económica (Anisi 1995, p. 70).

\section{El consenso keynesiano. Su visión}

Si se continúa con la relación mundo real y teoría como contexto explicativo del pensamiento económico, se puede explicar la génesis de la propuesta keynesiana. Antes de la interpretación del mundo real desde la perspectiva keynesiana, la presencia del enfoque marshalliano explicaba dicha relación a través de la premisa determinación de los precios, pero después de la segunda guerra mundial aparecen los ciclos de negocios ya no presente en Marshall (Heilbroner y Milberg, 1998, p. 52), sin embargo, el ciclo de negocios no se constituiría en la visión del pensamiento económico y se plantea desde Keynes la determinación de la demanda agregada ${ }^{17}$ que se convertiría en el interés común del pen-

17 No obstante se da continuidad al sistema de precios 
samiento económico y pilar para acercar la teoría al mundo real (Heilbroner y Milberg, 1998, p. 52), es precisamente esta situación lo que legitimaría la constitución del Estado de Bienestar:

"...el análisis económico debía ser reconstruido para llevar los problemas agregativos a corto plazo al centro de la escena. Las cuestiones microeconómicas, en torno a las cuales se había organizado la tradición neoclásica, fueron dadas de lado" (Barber, 1984, p. 218).

La propuesta de Keynes aporta al análisis económico el papel de la incertidumbre y las expectativas. Con ello, se establece una nueva forma en la solución de los problemas de orden económico y la mediación política del Estado ${ }^{18}$ :

“...John Maynard Keynes y el grueso de los economistas del siglo XX, no tuvieron ni siquiera la preocupación ante la ampliación del tamaño del Estado: más aún, la recomendaron como la mejor forma de resolver los problemas económicos" (Rodriguez Braun, 2002, p. 20).

\section{La crisis del pacto keynesiano.}

La economía keynesiana contribuyó a la estabilidad del sistema capitalista, incorporando una serie de mecanismos $^{19}$ que le permitió al sistema hacer converger intereses aparentemente contrapuestos (intereses del colectivo humano y del Estado) e incorporar te- rritorios e individuos a la lógica del valor. Se consolidó de esta forma el Estado de Bienestar (pacto keynesiano)

"aquel que permitió, en Occidente, tras la Segunda Guerra Mundial, la interrelación y apoyo mutuo entre el capitalismo desarrollado y lo que hemos venido a denominar Estado de Bienestar. El resultado de ese pacto...permitió el diseño de una política económica donde quedaba garantizado el pleno empleo del capital y el trabajo, se ofrecían altos tipos de beneficio, y se establecía una red de seguridad para los ciudadanos en general y para los asalariados en particular"(Anisi, 1989, p. 241).

Son varios los argumentos que pueden explicar el deterioro del objeto funcional en la relación teoría-mundo real (visión). Uno de ellos, radica en que el enfoque keynesiano fue incapaz de mostrar un cuerpo teórico riguroso de la inflación.

Otro de los argumentos de orden analítico es la imposibilidad de la economía keynesiana para asumir el concepto de estanflación en su estructura (Heilbroner y Milberg, 1998, p. 82). Se traduce lo anterior en el sentido de que el modelo no pronosticó la reestructuración y desestructuración del ámbito económico y político ${ }^{20}$.

Otro más que podría explicar la crisis del keynesianismo es la manera

18 El Estado de Bienestar fue el centro gravitacional de la racionalidad (eficiencia económica e igualdad social).

19 Para el Estado la redistribución es una necesidad económica, mediante el cual se garantiza el crecimiento. Es preciso políticamente este análisis para garantizar la cohesión social.

$20 \mathrm{Al}$ respecto $\mathrm{O}^{\prime}$ connor (1972, p. 19-20) esgrime que los fallos son reflejos del mismo sistema. Muestra que la intervención estatal en la sociedad capitalista está colmado de contradicciones. 
como abordó el dinero. La política fiscal no tuvo la capacidad predictiva para frenar la inflación.

La crisis del keynesianismo, si bien puede explicarse en términos del carácter analítico, podría ser explicada, además, por el refinamiento que realiza el sistema capitalista en los instrumentos que garantizan el proceso de acumulación de capital, en donde las reglas de juego en la relación EstadoSociedad cambian para mantener la regla fundante (el valor económico).

\section{La nueva visión del pensamiento} económico. Una revisión critica.

Con respecto al declive del modelo keynesiano hay un consenso generalizado en los círculos intelectuales, sin embargo, la nueva tarea con respecto a los posibles modelos radica en la manera como podrían establecerse la relación del mundo real y la teoría (visión). Emergió el monetarismo pero este fracasó como expresión teórica (Heilbroner y Milberg, 1998, p. 100103). Recuérdese que el monetarismo fue presentado como la propuesta de la derecha contra el pacto keynesiano, alrededor de la asistencia pública y la preferencia por los impuestos como pilares del Estado de Bienestar.

Una de la teoría que surgió como posible escuela que sustituía al keynesianismo fueron las expectativas racionales, la cual se apoyó en una menor fragilidad analítica (Mankiw, 1990)

Las expectativas racionales ("nueva macroeconomía clásica" o "monetarismo II"), fue una escuela apoyada en apreciaciones certeras del futuro, se sustentan en el empleo por parte de las unidades económicas de la información disponible en el mercado, de esta manera, predicen racionalmente el impacto de las decisiones estatales y actúan de acuerdo a las expectativas que han construido (Ekelund y Hebert, 1992, p. 585). En la línea que se ha venido presentando el artículo, es importante resaltar, que las expectativas racionales deben interpretarse desde la posibilidad que tiene en términos de su carácter comprensivo de la realidad.

Quedan por presentar dos escuelas, la neoclásica y la neokeynesiana. La escuela neoclásica encaja el suelo macroeconómico sobre premisas macroeconómicas (preferencias y tecnología) con el objeto de hacer inmune su estructura a la mediación política del Estado (Heilbroner y Milberg, 1998 p. 111). El asumir las premisas de merado autorregulado y optimalidad social, deja por fuera los conflictos entre los hombres y se presenta como contradicción debido a que la ciencia economía aborda los problemas sociales, al respecto (Heilbroner y Milberg, 1998, p. 114) exponen:

"al formalizar y situar al individuo -el supuesto agente representativo- en el centro del análisis, los neoclásicos han eliminado todos los aspectos sociales del comportamiento como el poder, el compromiso y los valores. A efectos prácticos, han eliminado lo individual o el mismo individuo. Una insistencia en la sociabilidad de los agentes implica un enfoque muy distinto de la economía"

Con respecto a la escuela neokeynesiana según Heilbroner y Milberg 
(1998, p. 118), esta no reduce el papel activo de la administración pública:

"lo que convierte en "nuevos" a los modelos neokeynesianos es que se construyen sobre el fundamento explícito de comportamientos basados en las expectativas racionales y guiados por la realización de toda oportunidad existente para maximizar el bienestar individual".

El propósito es mostrar que la maximización no es condición suficiente para generar resultados del orden neoclásico si el mercado no actúa como asignador eficiente de recursos. Siguiendo a Heilbroner y Milberg (1998, p. 119) se puede observar que la escuela neokeynesiana más que garantizar la relación mundo real-teoría, pone su acento en dar respuesta al argumento neoclásico de la imposibilidad de desempleo involuntario en el libre mercado. Se convierte así la escuela neokeynesiana en una postura constestaria al modelo neoclásico, en lugar de ocupar un espacio en la construcción de una nueva visión económica.

Al margen de esta critica, hay una consideración que acerca a esta escuela a los neoclásicos y monetaristas, y es la presencia de su enfoque desde la oferta, contrario a la propuesta de la demanda de los keynesianos. Así se pone de relieve un interés en las fallas de mercado:

"la economía neokeynesiana se parece cada vez más a un juego jugado con y contra otros economistas -de cuyos resultados nada depende mucho excepto el prestigio académico- que a un asunto que deba desarrollarse (...) en el mundo real" (Heilbroner y Milberg (1998, p. 121-122).

Lo que se venido tratando hasta estos momentos es la presentación de las distintas escuelas y su relación con las condiciones de realidad, es decir, lo que se ha denominado "visión". Para que la visión sea un hecho, según Heilbroner y Milberg (1998, p. 124), los economistas examinan tres elementos categóricos.

El primero de ellos consiste en el cumplimiento de las exigencias de la sociedad en lo que concierne a la explicación de los problemas de carácter social y económico. El segundo elemento es la incorporación de la política en la explicación de hechos económicos concretos y por último, considerar que la defensa del mercado es una acción política.

\section{Una explicación del fracaso actual en la relación teoría-mundo real}

Se ha señalado de manera sucinta que la configuración del Estado de Bienestar y consolidación del mismo, obedece fundamentalmente al soporte teórico e ideológico de corte keynesiano, cuyo objeto fue someter los problemas económicos a la disciplina de orden político, y además mantener muy estrecha la relación entre la teoría y las condiciones de realidad social ${ }^{21}$.

En el modelo keynesiano se reconoce que la intervención del Estado

21 Hay que aclarar que la política en la escuela keynesiana no se instaura en el momento inicial de la relación social sino que se coloca después. De esta manera se considera entonces que la política está a un lado de las relaciones de poder. 
en el proceso económico tiene como objetivo la corrección de las imperfecciones económicas y sociales que son generadas no mecánicamente. El Estado debe establecer las condiciones adecuadas para que el aparato productivo sea próspero ${ }^{22}$. Pero no le limita al mercado que se constituye en el principio rector de la realidad. El modelo Keynesiano de esta forma rompe con las escuelas anteriores. Esta situación podría explicar el declive del keynesianismo ya que las nuevas escuelas imponen como única forma de relación social al mercado (ley natural); por consecuencia estas escuelas tratan la investigación económica por fuera del sistema capitalista y la ubican en el mercado $^{23}$. La comprensión de los fenómenos sociales a partir de las leyes naturales dificulta que las nuevas escuelas expliquen la realidad social y puedan consolidar la relación teoríamundo real que es el terreno de legitimidad del Estado de Bienestar ${ }^{24}$.

Otra dificultad para que las nuevas escuelas reemplacen la keynesia- na, es el divorcio que se presenta entre ciencia económica y sistema capitalista $^{25}$ (Heilbroner y Milberg, 1998, p. 135), pues la ciencia económica concebida en la actualidad se ubica de manera inexorable en el mercado, y no se explora la relación ciencia económica y sistema capitalista:

"en realidad el significado de 'económico' sería ininteligible fuera del capitalismo (...) La psicología, la sociología y la política no incluyen al desempleo, o al crecimiento desigual, en sus preocupaciones conceptuales o analíticas. Lo que quiere decir que existen aspectos de un orden capitalista que no se pueden entender sin la economía; o por darle la vuelta, quiere decir que la economía no se puede aprender o utilizar sin hablar de capitalismo" (Heilbroner y Milberg 1998, p. 144).

Para terminar, una de las dificultades que han tenido las escuelas económicas hoy en día es poder garantizar la relación teoría-mundo real desde la misma configuración del sistema. A medida que se aproxima el siglo XXI,

22 Galbraith (1974, p. 25) argumenta que cuanto más capital se compromete en la empresa será tanto más arriesgado fiarse de las respuestas espontáneas de las necesidades del consumidor.

23 A diferencia de Keynes, hoy se ha vuelto a la tradicional ortodoxia económica; primero se crece y luego se distribuye. Habría que agregar que hoy en día las leyes del mercado es una condición no sólo del contexto mercantil sino del orden político. La organización de las relaciones sociales no dependen de las relaciones de poder sino que son el resultado de una premisa técnica (economicismo). Así la teoría económica establece relación con una sociedad que ha excluido sus variables que no competen a las del mercado.

24 Galbraith (1992) hace hincapié en las condiciones sociales precarias dentro de la economía americana, lo que se traduce en un descontento social creciente.

25 Recuérdese que el capitalismo como organización social es de carácter sociopolítico, legitima el mercado y la presencia de espacios públicos y privados. 
la desaparición de un corpus plausible de la teoría económica se torna cada vez más costoso en términos de establecer los fundamentos para políticas eficaces para mitigar los retos futuros (Heilbroner y Milberg (1998; pp. 149-150).

\section{Conclusiones}

Hasta bien entrado el siglo XX, la relación entre las ciencias económicas y el Estado de Bienestar posibilitaron el reconocimiento de la actividad económica y la seguridad social. Entrados los ochenta, la profundización de las condiciones de precariedad social reflejada en la creciente desigualdad en la distribución del ingreso y en la constitución de una sociedad excluyente, han ocasionado la pérdida de legitimidad por parte de la sociedad hacia el Estado de Bienestar.

La deslegitimación del Estado de Bienestar podría estar explicada a la largo de las transformaciones del pensamiento económico (responsable de imprimir la base teórica). Los cambios en el horizonte de cada una de las recientes escuelas (expost a la Keynesiana) consistente en sustituir el objetivo de la organización social por la del mercado, esto ha ocasionado que las escuelas dejen de lado el componente humanista del sistema capitalista como construcción histórica.

En este sentido, hay que considerar que el EB fue constituido en sus comienzos, con el objeto de que los ciudadanos, sin distingo de estrato social, pudiesen responder adecuadamente a los intereses del Estado a través de las garantías de la condiciones para que actuaran como individuos de mercado y constitutivos de Estado. Hoy por hoy, este propósito se ha ido desvaneciendo a medida que las escuelas económicas intentan explicar problemas del mercado y deja por fuera los problemas sociales del sistema capitalista como organización social.

\section{Bibliografía}

ANISI, D., (1992). Jerarquía, Mercado, Valores. Una Reflexión Económica sobre el Poder, Alianza Editorial, Madrid. ASHFORD, D., (1989). La aparición de los Estados de Bienestar, traducción, B. Gimeno, Ministerio de Trabajo y Seguridad Social, Madrid.

BARBER, B., (1984), Strong Democracy. Participatory Politics for a New Age, University of California Press, Berkeley.

BAUMAN, Z., (2002) Modernidad líquida. Fondo de Cultura Económica, México.

CASTELLS, A y BOSCH, N., (1998). El futuro del Estado de Bienestar, Civitas, Madrid.

BANDRÉS, E., El Estado de bienestar en España: entre los logros del pasado y la oportunidad de las reformas. Departamento de Estructura e Historia Económica y Economía Pública, Zaragoza, Universidad de Zaragoza. s/f.

DOLLEANS. E., (1969). Historia del Movimiento Obrero. Ed. ZYX. Madrid.

EKELUND, R. y HEBERT, R., (1992). Historia de la teoría económica y de su método. Mc Graw Hill, Madrid. 
GARCIA-PELAYO, M., (1981). El Estado social y sus implicaciones. En J. Subirats y P. Vilanova (comps.), La evolución del Estado en el pensamiento político, Editorial Pretel, Barcelona.

GALBRAITH, J. K., (1974). El Nuevo Estado Industrial, Ariel, Espulgues.

GALBRAITH, J. K., (1992). La Cultura de la satisfacción.

GÓMEZ DE PEDRO, M.E., (2002). El Estado del bienestar. Presupuestos éti$\cos y$ políticos, Fundación universitaria Española, Madrid.

GONZÁLEZ-SEARA, L., (2000). Antecedentes y fundamentos teóricos y doctrinales del Estado de Bienestar. En Las estructuras del Bienestar en Europa, Editorial Civitas, Madrid.

GUERRERO, D., (1997) Historia del Pensamiento Económico Heterodoxo. Trotta editorial, Madrid.

HAYEK, F, A, V., (1985) Direito, legislação e libertade. Vol. II. Visao, São Paulo.

HEILBRONER y MILBERG., (1998) $L a$ crisis de visión en el pensamiento económico moderno. Paidós, Barcelona.

KEYNES, J., (1991). Teoría general de la ocupación, interés y el dinero, Fondo de Cultura Económica, traducción Hornedo, E. Madrid.

MISRHA, R., (1992). El Estado de Bienestar en crisis, Ministerio de Trabajo y Seguridad Social, Madrid.

MISRHA, R., (1993). El Estado del Bienestar en la sociedad capitalista, Ministerio de Asuntos Sociales, Madrid.

MUÑOZ DE BUSTILLO, R., (1989). Crisis el y futuro del estado del bienestar, Alianza, Madrid.

MUÑOZ DE BUSTILLO, R., (2000). El Estado de Bienestar en el cambio del siglo, Alianza editorial, Madrid.

MUÑOZ DE BUSTILLO, R.; BONETE, R., (2000). Introducción a la Unión
Europea: un análisis desde la economía, 2a. Edición, Alianza Editorial, Madrid.

MUÑOZ, S.; GARCÍA, J. L.; GONZÁLEZ, L.,(directores) (2000). Estado de Bienestar y Crecimiento económico, en Las estructuras del Bienestar en Europa, Editorial Civitas, Madrid.

NAVARRO, V., (1996). Neoliberalimo, desempleo, empleo y Estado de Bienestar, en Revista Sistema $N^{o} 134$, Septiembre 1996.

NAVARRO, V., (1997). Neoliberalismo y Estado del Bienestar, Ariel, Barcelona.

NAVARRO, V., (2000). Globalización económica, público de poder y Estado, Ariel, Madrid, 2000.

O'CONNOR, J., (1972) The fiscal crisis of the State, St. Martin's Press, New York.

LOJKINE, J., (1977) O Estado capitalista e a questão urbana. Ed. Martins Fontes, São Paulo.

MISSES, L, V., (1947) Le government omnipotent, Libraire de Médecis, París.

PETERSEN, W., La población. Madrid, Tecnos, s/f.

POLANYI, K., (1989) La gran transformación. Madrid, La piqueta.

RODRIGUEZ-BRAUN, C., (2002) Grandes economistas. Editorial Pirámide.

TREVELYAN, G. M., (1946) Historia social de Inglaterra. Fondo de Cultura Económica, México.

VELARDE, J., (1996). Hacia otra Economía Española, Espasa Editorial, Madrid.

VELARDE, J., (1994). Consideraciones sobre la crisis del Estado de Bienestar, En Revista del Instituto de Estudios Económicos y Empresariales, Madrid, Ministerio de Trabajo y Seguridad Social.

VILLARESPE, V., (2002) Pobreza, teoría e historia, México, Instituto de Inves- 
tigaciones Económicas-UNAM/Casa Juan Pablos.

VILLARESPE, V., (2001) Solidaridad: beneficencia y programas (pasado y presente del tratamiento de la pobreza en México), México, Instituto de In- vestigaciones Económicas-UNAM/ Miguel Ángel Porrúa, Colección. Textos breves de Economía.

WEBB, S, y WEBB, B., (1990) Historia del sindicalismo, 1660-1920, Madrid, Ministerio de Trabajo y Seguridad Social. 


\title{
RESÚMENES Y ABSTRACTS
}

\section{LOS VALORES EN LAS FAMILIAS EMPRESARIAS COLOMBIANAS}

\author{
Ernesto Barrera Duque
}

\section{RESUMEN}

Este artículo, a partir de una investigación cualitativa y práctica, propone un modelo general de análisis para los valores en las dinámicas operativas de las familias empresarias colombianas. En el cuerpo del texto, se identifican los valores vigentes, estableciéndose su contenido y jerarquía, en función de los resultados del trabajo de campo.

PALABRAS CLAVE: Familia empresaria, empresa familiar, valores.

\begin{abstract}
This article, from a qualitative and practical research, proposes a general model of analysis for the dynamic values in operative the families of the Colombian industralists. In the body of the text, the effective values are identified, settling down their content and hierarchy, based on the results of the work of field.
\end{abstract}

KEY WORDS: Family entrepreneur, familiar company, values. 


\title{
ANÁLISIS DEL DISEÑO DE LOS CONTRATOS EN EL PROCESO DE SUBCONTRATACIÓN DE LAS ORGANIZACIONES PÚBLICAS, EL CASO DE EMCALI EICE
}

\author{
Henry Caicedo Asprilla
}

\begin{abstract}
RESUMEN
Éste documento utiliza las categorías de la economía institucional para evaluar el impacto que tiene sobre las organizaciones públicas el proceso de outsourcing o subcontratación. Aquí se plantea el interrogante de hasta dónde es posible alcanzar la eficiencia de una organización pública cuando el proceso de subcontratación está determinado por contratos intrínsecamente incompletos. A partir de la información de una muestra de 198 contratos que Emcali firmó con proveedores entre los años 2001 y 2004, se halló que 128 estaban mal diseñados y sólo 70 cumplían con el requisito del buen diseño económico; este hallazgo permite verificar la presunción de la teoría económica institucional que afirma que aunque los contratos estén diseñados conforme a la ley, estos presentan fallas en el diseño económico y por consiguiente la empresa experimenta costos de transacción. En este docu-
\end{abstract}

mento se evidencia que en el caso concreto de Emcali existen dos variables que clasifican los contratos entre bien y mal diseñados: el valor de la inversión y el índice de riesgo de ejecución del contrato; mientras que las variables duración del contrato, el riesgo legal, empresas en licitación, garantías y pólizas de aseguramiento, consideradas fundamentales en el diseño contractual de una transacción no cumplen con el propósito de corregir las anomalías en los contratos. En este trabajo se avanza hasta plantear que existe una conexión entre el mal diseño de los contratos y las dificultades financieras y administrativas de Emcali, que llevó a la empresa a la crisis más grande de su historia.

PALABRAS CLAVE: Organizaciones públicas, outsourcing, análisis de los contratos, economía institucional, árboles de decisión. 


\section{ABSTRACT}

This paper uses the categories of the institutional economy to evaluate the impact that has on the public organizations the process of outsourcing or subhiring. The principal question is: until where considers it is possible to reach the efficiency of a public organization when the subhiring process is determined by intrinsically incomplete contracts. From the information of a sample of 198 contracts that Emcali signed whit suppliers between years 2001 and 2004, one was that 128 badly were designed and only 70 fulfilled the requirement of the good economic design, this finding allows to verify the economic presumption of the institutional theory that it affirms that although the contracts are designed according to the law, these contracts present faults in the economic design and therefore the company experiences transaction costs. This document evidences that in the Emcali's case there are two indicator that classify contracts between good and badly designed: the value of the investment and the index of risk of execution of the contract, whereas the indicators duration of the contact, the legal risk, companies in licitation, guarantees and insurance policies, were considered fundamental in the contractual design of a transaction do not correct the anomalies of the contacts. In this paper one advances when a connection is established between the wrong design of contracts and the financial and administrative difficulties of Emcali, that led the company to the greatest crisis of its history.

KEY WORDS: Public organizations, outsourcing, analysis of contracts, institutional economy, decition trees. 


\title{
¿DESDE UNA INGENIERÍA DEL ALMA HACIA UN "MANAGEMENT" DEL ESPÍRITU? ACERCA DE LA NOCIÓN DE PSICOLOGÍA EN EL DISCURSO ADMINISTRATIVO Y ORGANIZACIONAL
}

\author{
Héctor Bermúdez
}

\section{RESUMEN}

Este ensayo busca un acercamiento a la genealogía de lo que se reconoce en la administración como las "Ciencias del Comportamiento" y una crítica a la relación entre el pensamiento administrativo y aquella corriente particular de la psicología que ha sido incorporada a éste: lo que comúnmente se conoce como la psicología industrial pero que bien podría nombrarse como el conductismo organizacional.

Cabe decirse también, que, por el trabajo de consultoría especializada en Gestión Humana del autor, y por la docencia tanto en cursos de MBA para ejecutivos, como en programas para especialistas en administración de personal, fue posible articular esa experiencia de campo, con lo inferido del análisis teórico de la revisión documental. Así entonces, este trabajo busca apoyar la línea de investigación en estudios sobre la Gestión Humana.

PALABRAS CLAVE: Administración, Conductismo Organizacional, Psicoanálisis, Psicología Industrial, Organizaciones.

\begin{abstract}
This test looks for an approach of the genealogy of which a critic is recognized in the administration like "Sciences of the Behaviour" on the other hand, the article explain the relation between the administrative thought and that particular current of the psychology that has been incorporated to this one. This challenge is commonly known like the industrial psychology but that could well name like the organizational conductism.

It is possible also to say itself, that, by the work of consultancy specialized in Human Management of the author, and by teaching as much in courses of MBA for executives, like in programs for specialists in personnel administration, it was possible to articulate that experience of field, with the inferred thing of the theoretical analysis of the documentary revision. Then, this work looks for to support the line of research in studies on the Human Management.
\end{abstract}

KEY WORDS: Administration, Organizational Conductism, Psychoanalysis, Industrial Psychology, Organizations. 


\title{
LOS DESAFÍOS DEL DISEÑO ORGANIZACIONAL E INSTITUCIONAL EN UN MEDIO AMBIENTE GLOBALIZADOR
}

\author{
José G. Vargas Hernández
}

\section{RESUMEN}

Este trabajo tiene por objetivo analizar los desafíos de diseño organizacional e institucional en un medio ambiente globalizador. Este análisis se inicia a partir de las consideraciones de los procesos de globalización económica en la emergencia de las nuevas asimetrías del poder y su impacto en los procesos de cambio organizacional, institucional, del Estado y las políticas públicas y el diseño intergubernamental. La información y el conocimiento son insumos de las comunidades de aprendizaje y para la formación de redes. Una característica de los arreglos institucionales y nuevas formas organizacionales es la creación del poder en los individuos (empowerment)

PALABRAS CLAVE: Asimetrías del poder, diseño institucional, diseño organizacional, globalización

\begin{abstract}
This work must by objective analyze the challenges of organizational and institutional design in global environment. This analysis begins from the considerations of the processes of economic globalization in the emergency of the new asymmetries of the power and its impact in the processes of organizational, institutional change, of the State and the public policies and the intergovernment design. The information and the knowledge are raw material of the learning communities for the formation of networks. A characteristic of the institutional adjustments and new organizationals forms is the creation of the power in the individuals (empowerment)
\end{abstract}

KEY WORDS: Asymmetries of the power, institutional design, organizational design, globalization. 


\title{
LA REFORMA DEL ESTADO EN COLOMBIA: ENTRE EL AJUSTE FISCAL Y FORTALECIMIENTO DE LA PARTICIPACIÓN CIUDADANA
}

\author{
Rosalía Correa Young
}

\begin{abstract}
RESUMEN
La reforma del Estado en Colombia y la descentralización, desde que se aprobó el Acto legislativo 01 de 1986, pasando por los acuerdos institucionales, plasmados en la Constitución de 1991 y referidos a esta materia, así como la normatividad actual, son analizados en este artículo a la luz de los enfoques teóricos que estimularon un nuevo modelo de Estado, a partir de la percepción respecto del agotamiento del Estado de bienestar, interventor y asistencial.

El artículo explica la dinámica en Colombia frente a dos paradigmas: uno proveniente de la sociología y la ciencia política que define la reforma desde una perspectiva policéntrica y uno proveniente de la economía neoclásica que ofrece una perspectiva mercadocéntrica. La primera en-
\end{abstract}

cuentra fortalezas para la democratización, la equidad, la superación de los desequilibrios regionales y la inclusión social. La segunda, el posicionamiento de las lógicas del mercado con la reducción, o desaparición de la intervención del Estado en aquellas funciones que los privados pueden resolver más eficientemente. Uno y otro colocan a la sociedad civil en un protagonismo activo, sea político, en su relación con el Estado; sea económico, en su relación con el mercado, pero ambos la conciben como actor de primera línea.

PALABRAS CLAVE: Reforma del Estado, Descentralización, Democratización, Paradigma policéntrico, Paradigma mercadocéntrico, Ajuste Fiscal, Participación, Privatización 


\section{ABSTRACTS}

The reform of the state in Colombia and the decentralization, since legislative Act 01 of 1986 was approved, happening through the agreements institutional, shaped in the Constitution of 1991 and referred this matter, as well as the present normatividad, are analyzed in this article to the light of the theoretical approaches that stimulated a new model of state, from the perception respect to the exhaustion of the welfare state, auditor and healthcare.

The article explains dynamics in Colombia as opposed to two paradigms: one originating of sociology and political science that the reform defines from a policentric perspective and one originating of the neoclassic economy that offers a market perspective. First it finds strengths for the democratization, the fairness, the overcoming of the regional imbalances and the social inclusion. Second, the positioning of the logics of the market with the reduction, or disappearance of the intervention of the State in those functions that the prevailed ones can solve more efficiently. Both places to the civil society in an active protagonism, is politician, in their relation with the State; be economic, in his relation with the market, but both conceive it like actor of forward edge.

KEY WORDS: The reform of the State, Decentralization, Democratization, policentric Paradigm, market Paradigm, Fiscal Adjustment, Participation, Privatization. 


\section{TEORÍA ECONÓMICA Y ESTADO DEL BIENESTAR. UNA APROXIMACIÓN}

Fernando Salazar Silva

\section{RESUMEN}

El siguiente artículo realiza un tránsito por algunas posturas dentro de las ciencias económicas para discutir el EB y presentar alternativas concretas al mismo. El propósito de Keynes en su afán de hacer legitimar la configuración y consolidación del Estado de Bienestar, se discutirá en el contexto de las particularidades de carácter teórico económico. Si bien el keynesianismo pudo concretar desde las ciencias económicas las bases para acercar la teoría a la realidad social, no entendemos por qué otras escuelas no han logrado constituirse en la base teórica del Estado de Bienestar actual.

PALABRAS ClAVE: Estado de Bienestar, Pacto keynesiano, Ciencia económica, Monetarismo, Expectativas racionales, teoría Neoclásica, Neokeynesianismo.

\begin{abstract}
The following article makes a transit by some positions within economic sciences to discuss the EB and to present concrete alternatives to he himself. Keynes's intention in its eagerness to make legitimize to the configuration and consolidation of the Welfare State, will be discussed in the context of the particularities of economic theoretical character. Although the keynesianism could make specific from economic sciences the bases to approach the theory the social reality, we do not understand so that other schools have not managed to constitute themselves in the theoretical base of the Welfare State present.

KEY WORDS:

Welfare State, Keynesian Accord, Economic Science, Monetarism, Rational Expectations, Theorie Neoclassic, Neo-Keynesianism.
\end{abstract}




\title{
LA RESPONSABILIDAD CIVIL AUTOMOVILÍSTICA Y EL ASEGURAMIENTO OBLIGATORIO Y VOLUNTARIO: UN ESTUDIO EN EL DERECHO COLOMBIANO Y ESPAÑOL
}

\author{
Raquel Ceballos Molano
}

\begin{abstract}
RESUMEN
La accidentalidad vial en Colombia es una de las primeras causas de daños corporales y materiales en la población circulante y peatonal, que obliga, al menos en teoría, al propietario o conductor, a responder civilmente de sus consecuencias. Así, este trabajo de tesis plantea el problema de la tipicidad como "actividad peligrosa" y la dispersión normativa derivada de la responsabilidad civil automovilística y los mecanismos de reparación a través del seguro voluntario y obligatorio del automóvil, consagrada en la legislación Civil, Penal, Comercial, Código Nacional de Tránsito, y en Decretos y Resoluciones administrativas, además de la doctrina científica y jurisprudencial, lo que evidencia que el legislativo colombiano no ha prestado atención, desde el punto de vista técnico y jurídico a las políticas de Estado para armonizar en un solo estatuto la responsabilidad derivada de los accidentes de tránsito y su aseguramiento, como si ocurre en el derecho comparado, el ordenamiento jurídico europeo y español.
\end{abstract}

PALABRAS CLAVE: Accidente de transito, responsabilidad civil, seguro obligatorio, soat, seguro automoviles, daño, indemnizacion, reparacion integral.

\section{ABSTRACT}

The road crash in Colombia is one of the first causes of body and materials damages in the circulating and peatonal population, that forces, at least in theory, to the proprietor or conductor, to respond civilly of its consequences. Thus, this thesis work creates the problem of the type like "dangerous activity" and the normative dispersion derived from the automobile civil responsibility and the mechanisms of repair through voluntary and obligatory insurance of the automobile, consecrated in the Civil, Penal, Commercial legislation, National Code of Transit, and in Decrees and administrative Resolutions, in addition to the scientific and jurisprudencial doctrine, which evidence that the legislative Colombian has not paid attention, from the technical and legal point of view to the state policies to harmonize in a single statute the responsibility derived from the traffic accidents and its securing, as if happens in the compared right, the European and Spanish legal ordering.

KEY WORDS: Road accident, civil responsibility, surely obligatory, soat, surely automobiles, damage, indemnification, integral repair. 


\title{
ENVEJECIMIENTO DEL PERSONAL
}

\author{
Sergio Castrillón
}

\section{RESUMEN}

¿De que manera la literatura en ciencias administrativas ha abordado el estudio del envejecimiento del personal? ¿Cuáles son las razones que han motivado este interés en los últimos años? Cuáles son las lógicas subyacentes? Reconociendo las tendencias demográficas mundiales que reflejan el envejecimiento de la población; este artículo procura responder a esta preguntas, a partir de una revisión exhaustiva de las principales publicaciones que se han ocupado del tema en el contexto internacional. Nuestro propósito es aumentar la visibilidad de la problemática y contribuir al debate sugiriendo acciones que revaloricen su dimensión humana.

PALABRAS CLAVE: Administración - Envejecimiento del Personal - Recursos Humanos - Fuerza Laboral - Jubilación - Retiro - Competencias y Desempeño - Motivación - Entrenamiento

\section{ABSTRACT}

How Literature in administrative sciences is boarded the study of the ageing of the personnel? Which are the reasons that have motivated east interest in the last years? Which are the underlying logics? Recognizing world-wide demographic tendencies that reflect the ageing of the population; this article tries to respond to this questions, from an exhaustive revision of the main publications that have taken care of the subject in the international context. Our intention is to increase the visibility of the problematic one and to contribute to the debate being suggested actions that revalue their human dimension.

KEY WORDS: Administration Ageing of the Personnel - Human Resources -Labor Force - Retirement - Competitions and Performance - Motivation - Training 


\title{
RENDICIÓN DE CUENTAS: ESTRATEGIA PARA EL FORTALECIMIENTO DE LA DEMOCRACIA A TRAVÉS DE LA LIMITACIÓN DEL PODER
}

\author{
Carlos Wladimir Gómez Cárdena
}

\begin{abstract}
RESUMEN
Este artículo contiene como apuesta epistemológica la construcción de un modelo genérico de Rendición de Cuentas, el cual puede llegar a ser implementado en las diferentes entidades de naturaleza estatal. A partir de una elaboración teórico - conceptual de la Rendición de Cuentas y de una observación práctica de los primeros ejercicios de rendición de cuentas llevados a cabo en Colombia, se exploran diversas potencialidades e instrumentos que pueden llegar a fortalecer las democracias modernas a través fundamentalmente, de la limitación de los ejercicios de poder. En las democracias modernas son muchos los mecanismos formales de limitación del poder consignados en el conocido sistema de frenos y contrapesos, pero son muy pocos los mecanismos reales. La rendición de cuentas como práctica democrática puede presentarse como uno de estos últimos, en la medida en que la sociedad organizada explore y explote tales potencialidades.
\end{abstract}

PALABRAS CLAVE: Políticas Públicas / Administración Pública / Rendición de Cuentas / Democracia / Limitación del Poder.

\section{ABSTRACT}

This article contains like comely epistemological the construction of a generic model of Accountability, which can arrive to be implemented in the different organizations of state nature. Starting from a theoretical - conceptual elaboration of the Accountability and of a practical observation of the first exercises of Accountability carried out in Colombia, diverse potentialities and instruments are explored that can end up strengthening the modern democracies fundamentally to inclination, of the limitation of the exercises of power. In the modern democracies there are many formal mechanisms of limitation of the power consigned in the well-known system of check and balances, but there are very few real mechanisms. Accountability as democratic practice can be presented like one of these last ones, in the measure in that the organized society explores and exploit such potentialities.

KEY WORDS: Public Policies / Public Administration / Accountability / Democracy / Limitation of the Power. 